\title{
Optimized molecular reconstruction procedure combining hybrid reverse Monte Carlo and molecular dynamics
}

\author{
Colin Bousige, ${ }^{1,2}$ Alexandru Boţan, ${ }^{1,2}$ Franz-Josef Ulm, ${ }^{1}$ Roland J.-M. Pellenq, ${ }^{1,2,3}$ \\ and Benoît Coasne ${ }^{1,2, a)}$ \\ ${ }^{1}$ Department of Civil and Environmental Engineering, Massachusetts Institute of Technology, 77 Massachusetts \\ Avenue, Cambridge, Massachusetts 02139, USA \\ ${ }^{2}<M S E>^{2}$, UMI 3466 CNRS-MIT, 77 Massachusetts Avenue, Cambridge, Massachusetts 02139, USA \\ ${ }^{3}$ CINaM, CNRS/Aix Marseille Université, Campus de Luminy, 13288 Marseille Cedex 09, France
}

(Received 4 February 2015; accepted 3 March 2015; published online 19 March 2015)

\begin{abstract}
We report an efficient atom-scale reconstruction method that consists of combining the Hybrid Reverse Monte Carlo algorithm (HRMC) with Molecular Dynamics (MD) in the framework of a simulated annealing technique. In the spirit of the experimentally constrained molecular relaxation technique [Biswas et al., Phys. Rev. B 69, 195207 (2004)], this modified procedure offers a refined strategy in the field of reconstruction techniques, with special interest for heterogeneous and disordered solids such as amorphous porous materials. While the HRMC method generates physical structures, thanks to the use of energy penalties, the combination with MD makes the method at least one order of magnitude faster than HRMC simulations to obtain structures of similar quality. Furthermore, in order to ensure the transferability of this technique, we provide rational arguments to select the various input parameters such as the relative weight $\omega$ of the energy penalty with respect to the structure optimization. By applying the method to disordered porous carbons, we show that adsorption properties provide data to test the global texture of the reconstructed sample but are only weakly sensitive to the presence of defects. In contrast, the vibrational properties such as the phonon density of states are found to be very sensitive to the local structure of the sample. (C) 2015 AIP Publishing LLC. [http://dx.doi.org/10.1063/1.4914921]
\end{abstract}

\section{INTRODUCTION}

Disordered porous carbons are heterogeneous materials with pore sizes ranging from $\sim 0.5 \mathrm{~nm}$ to few nanometers. Their high surface area and small pore size make them interesting materials for phase separation, filtration, catalysis, etc. ${ }^{1}$ Natural porous carbons such as coals or kerogens in gas shales are also of interest for the energy industry. It is well recognized that the microstructure of these materials governs their adsorption properties. As a consequence, the synthesis of artificial materials with some given properties-or the prediction of these properties for natural materials difficultly accessible to experiments—requires a reliable determination of their internal structure at the atomic scale. These atomic structures can then be used in computer simulations in order to predict adsorption equilibrium and kinetics, vibrational spectra, mechanical properties, etc. ${ }^{2,3}$

A review of the pros and cons of the various techniques used for generating realistic atomic models of amorphous and porous materials has been recently published by Palmer and Gubbins. ${ }^{4}$ These techniques can be separated into two categories. The first one is made up of "construction" techniques and encompasses (i) analogue development, which consists of constructing an analogue of the sample using well-known building blocks and (ii) mimetic simulations,

a)Electronic mail: coasne@mit.edu which numerically mimick the synthesis process. While such mimetic techniques have been widely used for porous silica, ${ }^{2,5}$ they can also be used for porous carbons by employing molecular dynamics (MD) simulations with reactive force fields. This method, referred to as quenched molecular dynamics (QMD), ${ }^{4,6}$ can be scaled up in order to develop large atomistic models capturing both micro- and mesoporosities in porous carbons. On the other hand, no experimental constraint is used so that one has no control over the final structure apart from the quenching rate, and QMD naturally tends to produce graphitized structures.

The second category of techniques used to generate realistic models of amorphous porous materials consists of "reconstruction" techniques, i.e., techniques aiming at producing a model taking into account experimental constraints. In the past 25 years, many efforts have been devoted to the reconstruction of realistic atomic structures of amorphous materials from experimental measurements. In the reverse Monte Carlo (RMC) procedure introduced by McGreevy and Pusztai, ${ }^{7,8}$ the experimental constraint is a radial pair correlation function $G(r)$ obtained by x-ray or neutron diffraction. Following a Metropolis algorithm, ${ }^{9}$ an atom is randomly displaced at each step of the simulation, and the acceptance probability is determined using the root-mean square difference $\chi^{2}=\sum_{i}\left[G_{\text {simul }}(r)-G_{\text {exp }}(r)\right]^{2}$ between the simulated and experimental $G(r)$. This procedure is robust and accurate for systems with 2-body interactions for which the 
correspondence between the structure and the $G(r)$ function is unique. ${ }^{10}$ As a result, it has been used successfully to describe the structure of liquids. ${ }^{11}$ However, this procedure cannot be applied to more complex systems where many-body interactions have to be taken into account since its application generally leads to unrealistic liquid-like structures.

A way to ensure that the molecular models obtained with a RMC procedure present a realistic chemistry is to introduce additional constraints. In their reconstruction technique of porous carbons, Thomson and Gubbins ${ }^{12}$ constrained the structure to be composed of graphene-like building blocks. This constraint proved to be valuable for reproducing the properties of graphitic carbons but too restrictive for most porous carbons. Another solution, known as constrained reverse Monte Carlo (CRMC), consists of adding angular, coordination, and distance constraints to the acceptance probability of the RMC moves. This procedure was used in the case of vitreous silica ${ }^{13,14}$ and carbonaceous structures. ${ }^{15}$ In the specific case of carbonaceous systems, the CRMC procedure was able to produce structures containing a variety of typical features such as 5-, 6- and 7-membered rings. These porous structures were also found to successfully predict $\mathrm{Ar}$ and $\mathrm{N}_{2}$ heats of adsorption at $77 \mathrm{~K}$ in activated carbons. ${ }^{16}$ However, the CRMC models are highly dependent on the set of imposed constraints, which can lead to unphysical biases. Moreover, the CRMC structures still exhibit unrealistic features such as 3- and 4-membered rings, which are unstable upon further MD relaxation. ${ }^{17,18}$

A refined strategy to constrain a RMC simulation consists of using an energy constraint calculated using a reactive force field, as proposed by Opletal et al. ${ }^{19}$ This approach is referred to as hybrid reverse Monte Carlo (HRMC) since it combines an energetic term with an experimental constraint in the Metropolis acceptance probability. Gereben and Pusztai have recently proposed a method, similar to HRMC, which combines into the same acceptance probability a term corresponding to structure-refinement and a term corresponding to energy penalties (instead of using bond and angle constraints as in constrained reverse Monte Carlo). ${ }^{20}$ The application of $\mathrm{CRMC}^{15}$ and $\mathrm{HRMC}^{21,22}$ to the same sucrose coke has shown that the HRMC procedure generates structures that are more stable and contain a significantly lower proportion of 3- and 4-membered rings compared to the CRMC procedure. However, the sum of the structural and energetic constraints can lead to a rather small acceptance ratio in the simulation; the system being therefore prone to remain trapped in local configurational minima. To overcome this issue, a simulated annealing technique can be performed, where the temperature of the simulation is slowly decreased from a large initial temperature to explore a maximum of configurations by overcoming energy barriers between configurations. Unfortunately, the HRMC procedure becomes computationally very expensive for systems containing a few thousands of atoms.

In order to reduce the configuration space of acceptable structures produced by RMC, Biswas et al. ${ }^{23}$ have suggested a procedure referred to as Experimentally Constrained Molecular Relaxation (ECMR). This method consists of alternate RMC simulated annealings and MD quenches until conver- gence is reached. Considering that RMC is known to produce unphysical liquid-like structures ("unphysical” for systems more complex than atomic liquids), the combined use of RMC and MD was found to improve the quality of the structures by subjecting the system to a force field that imposes a set of physical constraints. Although similar, this MD-HRMC method differs from that by Biswas et al. because the use of HRMC instead of RMC allows generating more physical structures by including energy penalties. Moreover, in the spirit of the work by Biswas et al., the combination of MD and HRMC simulations improves the sampling of the phase space by restraining sampling to physical configurations (HRMC) while allowing their relaxation and a better exploration scheme (MD).

Of key importance to assess the quality and efficiency of reconstruction techniques combining energy and structure optimizations, the relative weight $\omega$ given to the energy penalty with respect to the structure optimization in HRMC has always been defined in an empirical way. Here, we provide a physical rationalization of this weighing parameter as well as other parameters used in such a MD-HRMC strategy. Such parametrization rules are important as they allow a greater transferability of HRMC-based procedures. In order to demonstrate the strengths of such combined MD-HRMC simulations, we compare the structures obtained using this method and those obtained using QMD. We show that the significant morphological and chemical disorders in amorphous porous carbons cannot be described using QMD simulations (the latter leading to energy minimized ordered solids close to graphitic materials since they are not constrained to reproduce the sample structural properties).

This article is organized in two sections. After describing the MD-HRMC procedure and comparing it to HRMC and QMD, we provide rational arguments to select the various parameters needed in the simulations in order to provide simple rules and guides for conducting hybrid reverse Monte Carlo simulations with various systems. We then discuss the validity of the models obtained with this method using various characterization tools accessible to both experiment and molecular simulation. In particular, we show that the usual texture test consisting of computing the adsorption properties is not sensitive to the presence of defects in the structure. We suggest a more sensitive test which consists of measuring the vibrational properties of the system such as its phonon density of states (DOS).

\section{SIMULATIONS}

\section{A. Carbon sample}

CS1000a is an activated sucrose coke: an initial coke, CS1000, is prepared by pyrolizing sucrose at $1000^{\circ} \mathrm{C}$ under nitrogen flow. The activated form of CS1000, CS1000a, is obtained by heating CS1000 at $850^{\circ} \mathrm{C}$ in an atmosphere of $\mathrm{CO}_{2}$ for $20 \mathrm{~h}^{21,24}$ In our HRMC simulations, we used the experimental measurement of $G(r)$ reported in Fig. 3(c) of Ref. 21 as the target function. As a general rule, the total scattering function $S(Q)$ should be preferred as target functions since $G(r)$ functions are subject to Fourier transform errors 
(mostly due to the finite range of $Q$ values in the experimental data). However, computing on-the-fly $S(Q)$ during reverse Monte Carlo simulations is computationally very expensive compared to $G(r)$. Moreover, considering that the simulated $S(Q)$ would necessarily be affected by spurious oscillations arising from the periodic boundary conditions of the system, $G(r)$ functions are usually selected as target functions to reconstruct atomic models of the material or structure under study. The simulation box, which is cubic with a size $25 \AA$, has a density of $0.722 \mathrm{~g} / \mathrm{cm}^{3}$ and a $\mathrm{H} / \mathrm{C}$ ratio of $0.091 .^{21}$ The system thus contains 561 carbon atoms and 51 hydrogen atoms.

\section{B. Hybrid reverse Monte Carlo method}

The RMC method by McGreevy and Pusztai ${ }^{7}$ uses the Metropolis algorithm ${ }^{9}$ to produce atomic models matching an experimental function $f_{\text {exp }}$-generally the structure factor $S_{\text {exp }}(Q)$ or its inverse Fourier transform $G_{\text {exp }}(r)$. The latter allows avoiding additional computation of a Fourier transform at each MC step. The agreement between the simulated structure and the experimental target is assessed using the function $\chi^{2}$,

$$
\chi^{2}=\sum_{i} \frac{\left(f_{\operatorname{sim}}\left(x_{i}\right)-f_{\exp }\left(x_{i}\right)\right)^{2}}{\sigma_{\exp }\left(x_{i}\right)^{2}},
$$

where the sum is performed over the discrete ensemble $\left\{x_{i}\right\}$ of experimental points. The experimental error $\sigma_{\text {exp }}\left(x_{i}\right)$ is usually taken to be a constant. At each step of the RMC simulation, the new configuration $j$ is obtained from the old configuration $i$ by randomly displacing an atom. The acceptance probability of this move is determined by

$$
P_{i \rightarrow j}^{a c c}=\min \left[1, e^{-\Delta \chi^{2} / T_{\chi}}\right],
$$

where $\Delta \chi^{2}=\chi_{j}^{2}-\chi_{i}^{2}$ is the variation of $\chi^{2}$ due to the MC move and $T_{\chi}$ a weighting parameter. A MC move such as $\Delta \chi^{2}<0$ is always accepted. On the other hand, a move such as $\Delta \chi^{2}>0$ is accepted with the probability $P_{i \rightarrow j}^{a c c}=e^{-\Delta \chi^{2} / T_{\chi}}$. In order to explore a very large set of configurations and to ensure that the final structure is independent of the initial structure, the simulation is performed following a simulated annealing procedure: the initial temperature $T_{\chi}^{(0)}$ is set to a large value for a number of steps until no significant change in the test function $\chi^{2}$ is observed (first block). The temperature of the block $n$ is then obtained by a progressive decrease from $T_{\chi}^{(0)}, T_{\chi}^{(n)}=T_{\chi}^{(0)} a^{n}$ with $\left.a \in\right] 0,1\left[\right.$, until final convergence of $\chi^{2}$ is achieved.

The extension of the RMC method to account for the atomic interactions is known as the HRMC. ${ }^{19}$ Following the formalism introduced in Ref. 21, the new acceptance probability is defined as

$$
P_{i \rightarrow j}^{a c c}(\text { carbon })=\min \left[1, \exp \left(-\frac{1}{T_{\chi}}\left(\Delta \chi^{2}+\frac{\Delta U}{\omega}\right)\right)\right],
$$

where $\Delta U=U_{j}-U_{i}$ is the change in the total configurational energy of the system between the configurations $i$ and $j$ and $T_{\mathcal{X}}$, the effective temperature of the simulation. Since hydrogen atoms do not contribute to scattering, the experimental $G(r)$

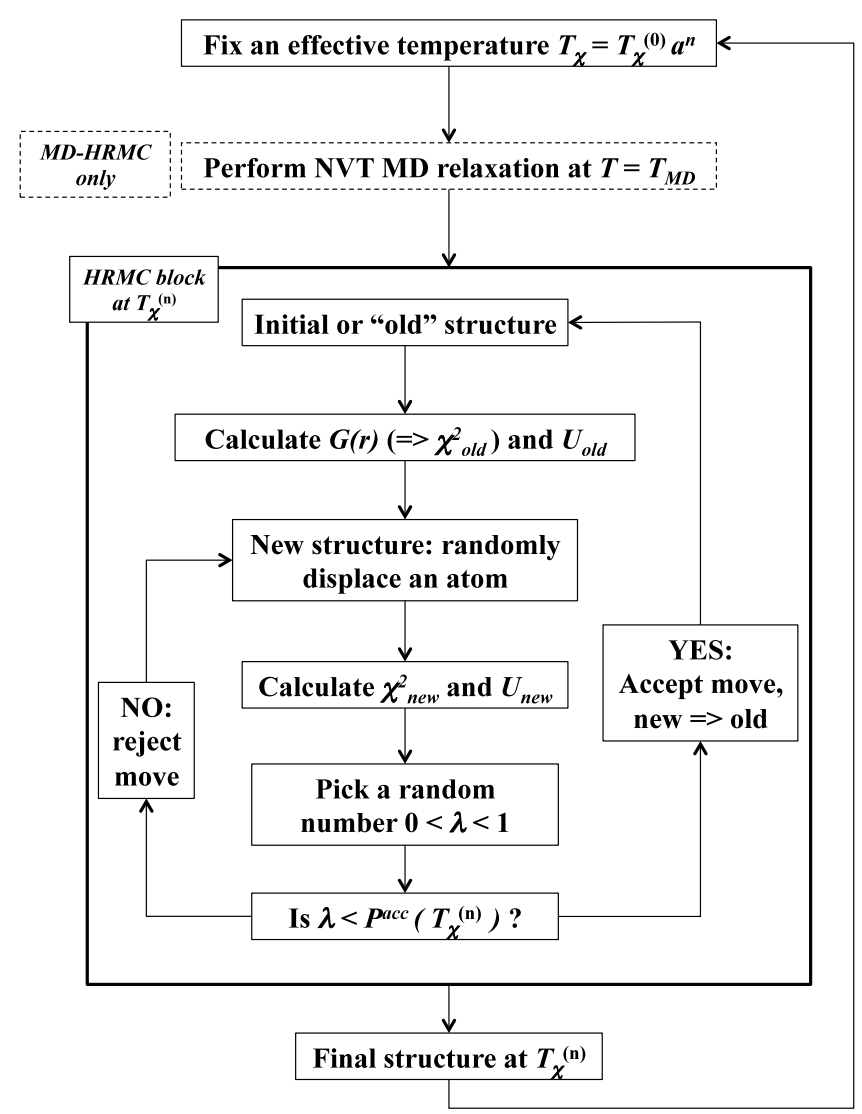

FIG. 1. Flow chart of the simulated annealing technique for HRMC and MD-HRMC simulations. MD-HRMC is the same as HRMC except for the additional MD relaxation block at temperature $T_{M D}$ (in the NVT ensemble) before the HRMC block (dashed lines).

function is a carbon-carbon pair correlation function only. Therefore, in the HRMC procedure, only carbon displacements verify Eq. (3), and hydrogen displacements obey the following regular Boltzmann acceptance probability:

$$
P_{i \rightarrow j}^{a c c}(\text { hydrogen })=\min \left[1, \exp \left(-\frac{\Delta U}{\omega T_{\chi}}\right)\right] .
$$

In Eqs. (3) and (4), $\omega$ is a parameter that weighs the RMC and MC contributions to the acceptance probability. If $\omega \rightarrow \infty$, the HRMC simulation is reduced to a RMC simulation, while it is reduced to a regular MC simulation if $\omega \rightarrow 0 . \omega$ is system dependent and one usually uses a trial and error technique to find a good compromise between $\chi^{2}$ and the number of highly constrained features such as 3- and 4-membered rings. We will discuss a method to rationalize the choice of $\omega$ in Sec. III. For a better visualization of the algorithm, a flow chart of the HRMC and simulated annealing procedure is presented in Fig. 1.

\section{MD-HRMC procedure}

The MD-HRMC procedure reported in this work combines the advantages of both HRMC and QMD by intercalating a block of $\mathrm{MD}$ relaxation at a temperature $T_{M D}$ between each HRMC block (Fig. 1). As in the case of HRMC, this strategy is combined with a simulated annealing technique. We expect from this procedure that MD relaxation at a sufficiently high 


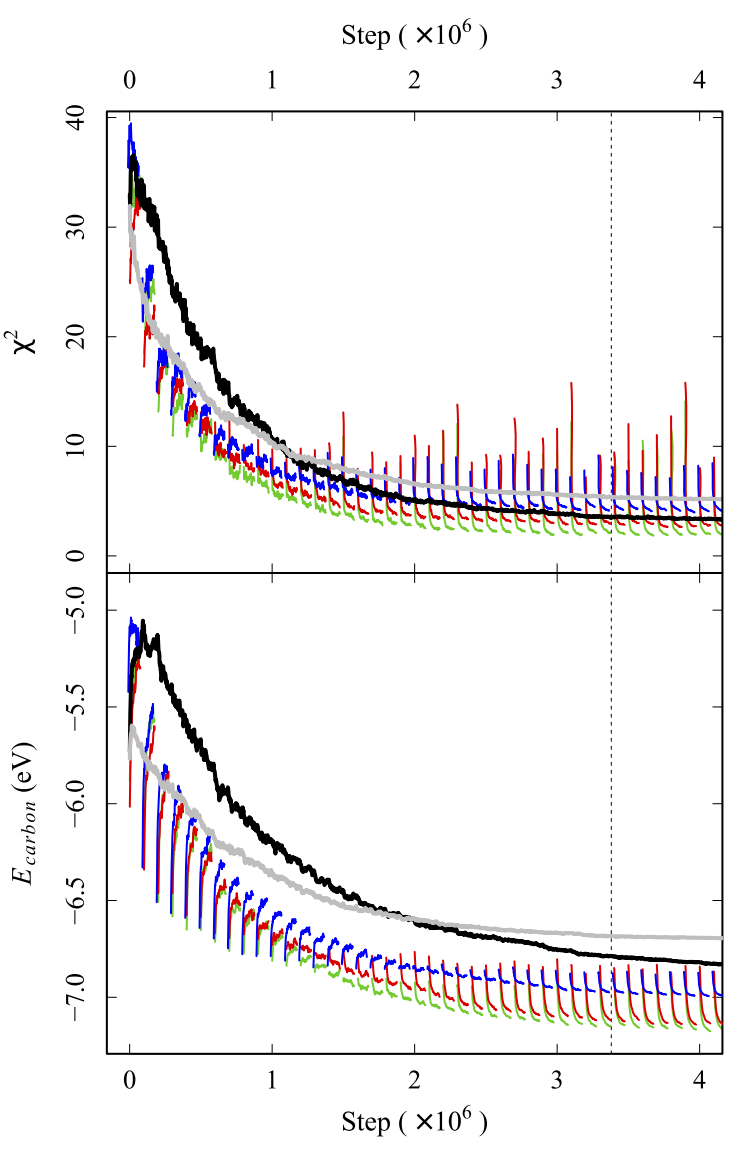

FIG. 2. $\chi^{2}$ (top) and energy per carbon atom $E_{\text {carbon }}$ (bottom) as a function of the number of steps for several HRMC and MD-HRMC procedures. HRMC simulations with $T_{\mathcal{X}}^{(0)}=10000 \mathrm{~K}$ (black) and $T_{\chi}^{(0)}=5000 \mathrm{~K}$ (gray), MD-HRMC simulations with $T_{M D}=2500 \mathrm{~K}$ (red), $T_{M D}=2000 \mathrm{~K}$ (green), and $T_{M D}=1800 \mathrm{~K}$ (blue). In all MD-HRMC simulations, $T_{\chi}^{(0)}=10000 \mathrm{~K}$. The vertical dashed line shows the end of the simulations at $T_{\mathcal{X}} \sim 300 \mathrm{~K}$ when $T_{\chi}^{(0)}=10000 \mathrm{~K}$.

temperature will allow the system to escape from local energy minima. In other words, by using MD-HRMC, we expect an enhanced efficiency in finding more stable configurations in a shorter computational time compared to HRMC.

We first discuss the choice of the various parameters in HRMC and MD-HRMC and compare these methods in terms of efficiency and quality. All the simulations below have been performed with the same initial random configuration (see Sec. II A) and the Reactive Empirical Bond Order (REBO) ${ }^{25}$ potential. The simulations were performed using a modified version of the LAMMPS $^{26}$ simulation package. A HRMC fix (LAMMPS' vocabulary for an operation that is applied to the system) was created as an extension to LAMMPS in order to easily switch from a HRMC simulation to a MD simulation. Except when specified otherwise, we have used $\omega=15 \mathrm{eV}$ as suggested by Jain et al. ${ }^{21}$ for CS1000a in order to compare our study with the literature. For the HRMC simulations, a block of HRMC moves at a given temperature $T_{\chi}$ contains $10^{5}$ steps (one step is defined as a move attempt, whether it is accepted or not). For MD-HRMC, a block contains 25000 MD steps at the temperature $T_{M D}$ in the NVT ensemble (time step $0.1 \mathrm{fs}$ ) followed by $75000 \mathrm{HRMC}$ steps at the temperature $T_{\chi}$.

Let us first discuss the HRMC simulations. The effective temperature $T_{\mathcal{X}}$ is a unitless parameter that verifies $\omega T_{\chi}$
$=k_{B} T$, where $T$ is the thermodynamical temperature of the simulation. For simplicity, in what follows, $T_{\chi}=10^{3} \mathrm{~K}$ means $T_{\chi}=\frac{k_{B}}{\omega} \times 10^{3} \mathrm{~K}$. Fig. 2 shows $\chi^{2}$ and the energy per carbon atom $E_{\text {carbon }}$ as a function of the number of steps for HRMC simulations with $T_{\chi}^{(0)}=10000 \mathrm{~K}$ (black lines) and $T_{\chi}^{(0)}=5000 \mathrm{~K}$ (gray lines). These data show that a large initial temperature allows exploring a larger region of the phase space and, hence, finding structures that are more stable and closer to the experimental target. In all cases, the decrease rate for the temperature was set to $a=0.9$ as suggested in Ref. 18, but a slower rate would probably result in further improvement of the final structures-a compromise has to be found between the quality of the model and the computation time.

In order to compare the MD-HRMC and HRMC procedures, all the MD-HRMC simulations were performed with $T_{\chi}^{(0)}=10^{4} \mathrm{~K}$. The only parameter that remains to be chosen is the MD relaxation temperature $T_{M D}$. Fig. 2 shows the evolution of MD-HRMC simulations for $T_{M D}=2500 \mathrm{~K}, 2000 \mathrm{~K}$, and $1800 \mathrm{~K}$. Oscillations are observed in the evolution of $\chi^{2}$ and $E_{\text {carbon. }}$. When $\omega T_{\chi} / k_{B}>T_{M D}, \chi^{2}$ and $E_{\text {carbon }}$ decrease upon MD while they increase upon HRMC. In contrast, after a large number of steps, i.e., when $\omega T_{\chi} / k_{B}<T_{M D}, \chi^{2}$ and $E_{\text {carbon }}$ increase upon MD while they decrease upon HRMC. For a given $T^{(0)}, \chi^{2}$ and $E_{\text {carbon }}$ in MD-HRMC are always lower than with HRMC. This shows that, at the end of the MD-HRMC simulations, the final structures are always more stable than with HRMC. Moreover, depending on $T_{M D}$, the final structure shows a lower $\chi^{2}$ than with HRMC. Finally, full convergence is reached for both $\chi^{2}$ and $E_{\text {carbon }}$ at the final temperature of $\sim 300 \mathrm{~K}$. In contrast, $\chi^{2}$ and $E_{\text {carbon }}$ have not fully converged in HRMC.

The temperature $T_{M D}$ must be chosen carefully. Too low temperatures make the atoms bond to each other very quickly so that the system does not have sufficient thermal energy to evolve into a more stable configuration. In contrast, too large temperatures force the system to escape from reasonably converged structures obtained during the HRMC blocks. This is illustrated in Fig. 2 and Table I showing that the structures obtained with $T_{M D}=1800 \mathrm{~K}$ and $2500 \mathrm{~K}$ lead to $\chi^{2}$ and $E_{\text {carbon }}$ larger than with $2000 \mathrm{~K}$. Moreover, the latter simulation leads to lower $\chi^{2}$ and $E_{\text {carbon }}$ than HRMC at $10000 \mathrm{~K}$. This result suggests that a temperature $T_{M D}=2000 \mathrm{~K}$ is optimal for MDHRMC simulations applied to porous carbons.

The refined quality of the MD-HRMC structures can be probed in Fig. 3 which shows their 2-fold-coordinated bond angle distribution for carbon atoms $(\mathrm{C}-\mathrm{C}-\mathrm{C}$ bond angle distribution). The distributions obtained using the different simulation techniques exhibit three principal features. (1) The

TABLE I. $E_{\text {carbon }}, \chi^{2}$ and number $N_{3}$ of 3 -membered rings in the final structures of the different simulations.

\begin{tabular}{lccr}
\hline \hline Simulation & $E_{\text {carbon }}(\mathrm{eV})$ & $\chi^{2}$ & $N_{3}$ \\
\hline HRMC 10000 K & -6.67 & 4.20 & 10 \\
HRMC 5000 K & -6.65 & 5.90 & 8 \\
MD-HRMC 2 500 K & -7.12 & 2.97 & 2 \\
MD-HRMC 2000 K & -7.15 & 2.08 & 2 \\
MD-HRMC 1 800 K & -7.08 & 3.06 & 4 \\
\hline \hline
\end{tabular}




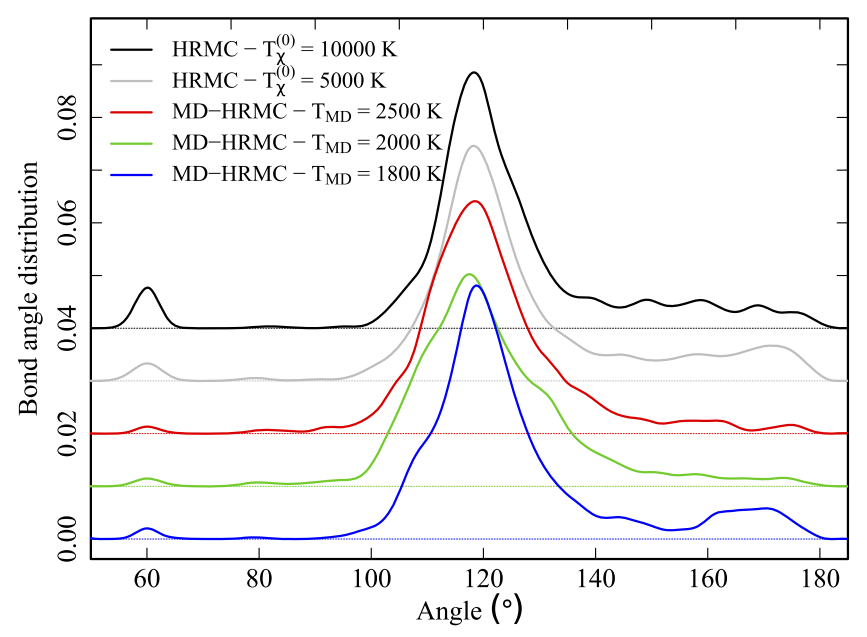

FIG. 3. C-C-C bond angle distribution for the structures obtained using HRMC and MD-HRMC procedures shown in Fig. 2. The distributions have been shifted up for the sake of clarity.

broad peak around $120^{\circ}$ with a shoulder around $110^{\circ}$ are representative of the $\mathrm{sp}^{2}$ and the $\mathrm{sp}^{3}$ carbon atoms, respectively. (2) The broad peak around $170^{\circ}$ is due to carbon atoms linked to each other in a chain with no hydrogen. This peak, which is pronounced in the MD-HRMC at $1800 \mathrm{~K}$ as well as in both HRMC simulations, illustrates the difficulty to relax local defects with this type of simulations because of insufficient thermally driven rearrangement. (3) Finally, both HRMC simulations generate structures with a non-negligible angle density at $60^{\circ}$, which corresponds to 3 -membered rings that are unfavorable and therefore unlikely to occur in real structures. In contrast, MD-HRMC significantly reduces the occurrence of these 3-membered rings (Table I). This result constitutes another argument in favor of the MD-HRMC method. Jain et al. ${ }^{21}$ have reported that the structures produced with HRMC do not present any 3-membered rings. However, these authors

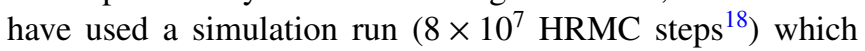
is much longer than the present HRMC simulations. We have found that MD-HRMC is at least one order of magnitude faster to reach structures of similar quality.

\section{DISCUSSION}

\section{A. Rational choice for $\omega$}

We now discuss how $\omega$ can be chosen to make MDHRMC transferable to other systems. The commonly used definition of $\chi^{2}$ given in Eq. (1) $)^{19,21,22}$ is extensive so that it depends on the number of experimental points used. The intensive definition

$$
\chi^{2}=\frac{\sum_{i}\left(f_{\operatorname{sim}}\left(x_{i}\right)-f_{\text {exp }}\left(x_{i}\right)\right)^{2}}{\sum_{i}\left(f_{\exp }\left(x_{i}\right)\right)^{2}}
$$

uses $\chi^{2}$-and thus $\omega$-that are independent of the number of points in the $G(r)$ function. In the same manner, one should use an intensive definition of the energy of the system, leading to the new acceptance probability:

$P_{i \rightarrow j}^{a c c}($ carbon $)=\min \left[1, \exp \left(-\frac{1}{T_{\chi}}\left(\Delta \chi^{2}+\frac{\Delta U}{N} \frac{1}{\tilde{\omega}}\right)\right)\right]$,

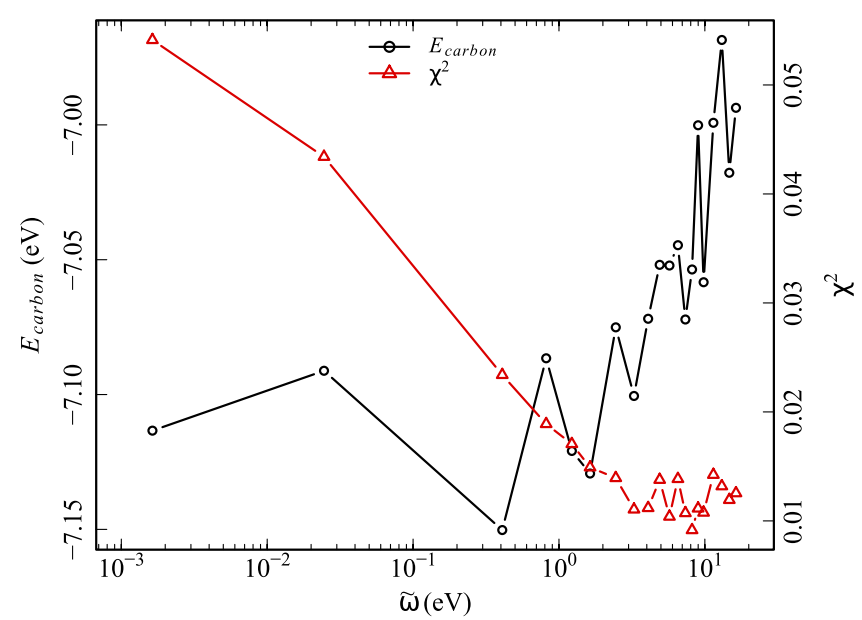

FIG. 4. Final $E_{\text {carbon }}$ and $\chi^{2}$ for various simulations as a function of $\tilde{\omega}$ $=\omega / N$. In these simulations, $\chi^{2}$ is defined by Eq. (5).

where $N$ is the total number of atoms and $\tilde{\omega}=\omega / N$. Fig. 4 shows the evolution of $E_{\text {carbon }}$ and $\chi^{2}$ as a function of $\tilde{\omega}$ used in MD-HRMC. $E_{\text {carbon }}$ increases while $\chi^{2}$ decreases upon increasing $\tilde{\omega}$. From these simulations, we can estimate that the optimal value of $\tilde{\omega}$ is about $7 \mathrm{eV}$, which is close to the final energy $E_{\text {carbon }}$ for these simulations. This shows that the expected final energy per carbon atom provides a reasonable starting value for $\tilde{\omega}$. In the case of highly hydrogenated systems, this definition is however flawed as the hydrogen atoms contribute to the energy but not to $\chi^{2}$. In order to correct for the additional energy of the hydrogen atoms, $\tilde{\omega}$ can be defined as

$$
\tilde{\omega}=\frac{N}{N_{\text {carbon }}} \times\left|E_{\text {carbon }}\right|,
$$

with $\left|E_{\text {carbon }}\right| \simeq 7 \mathrm{eV}$ as an initial guess. Such a value for the energy per carbon atom is system dependent and should be considered as a lower limit corresponding to the case where the hydrogen content is low. The definition in Eq. (7) with an energy per atom of $-7 \mathrm{eV}$ applied to CS1000a yields $\tilde{\omega}=7.6 \mathrm{eV}$. In the case of $\chi^{2}$ defined with Eq. (1) and the simulations of Sec. II C where $\sum_{i}\left(G_{\text {exp }}\left(x_{i}\right)\right)^{2} \simeq 228$, keeping the same $\Delta U / \Delta \chi^{2}$ ratio leads to $\omega \sim 20 \mathrm{eV}$. This value is close to that found by Jain et al. in their optimization procedure $(15 \mathrm{eV}) .{ }^{21}$

The definition above of the parameter $\omega$ provides a reasonable initial guess. This is supported by the fact that this definition allowed us to reconstruct very different amorphous carbonaceous structures (with different $\mathrm{H} / \mathrm{C}$ ratios) using MDHRMC. Of course, trial and error strategies can be used to further refine this parameter. However, as seen in Fig. 4, the use of MD relaxation steps ensures that the structures are physically stable and limits the impact of $\omega$ on the final structure (provided it was initially chosen according to our definition).

\section{B. Validation of the structures}

\section{Pair correlation function $G(r)$}

We now discuss the quality and stability of the structures obtained with MD-HRMC. Fig. 5(a) compares the experi- 

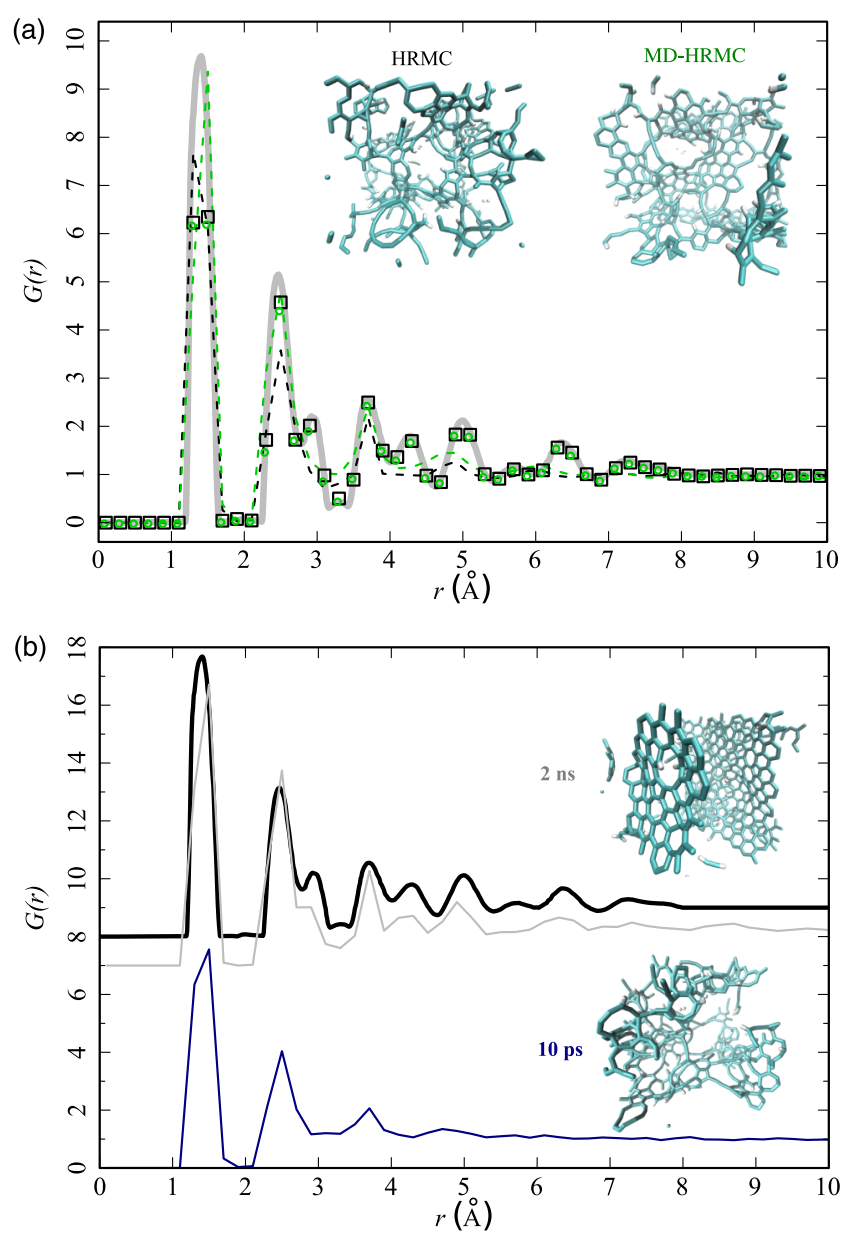

FIG. 5. (a) Experimental pair correlation function $G(r)$ (thick line, reproduced from Ref. 21) compared to the $G(r)$ computed from two of the structures obtained after HRMC (black squares) or MD-HRMC (green circles). Both structures were obtained using the $\omega$ parameter as defined in Sec. III A. Once relaxed $10 \mathrm{ps}$ in the NVE ensemble at $300 \mathrm{~K}$, both $G(r)$ evolve and long-range order are lost (dashed lines). (b) Experimental $G(r)$ (black line) compared to the $G(r)$ of structures obtained with QMD, where the system is quenched from $15000 \mathrm{~K}$ down to $300 \mathrm{~K}$ with various times. QMD with slow quenching leads to graphitized structures which are inconsistent with the known disordered structure of the experimental sample.

mental and simulated $G(r)$. After the MD-HRMC procedure, the simulated structures have been relaxed using MD simulations at $300 \mathrm{~K}$. Fig. 5(a) shows that, upon relaxation, the correlations after $4 \AA$ have almost disappeared - only the three first neighbor peaks at $1.4 \AA$, $2.5 \AA$, and $3.7 \AA$ remain. However, if the long-range order is lost upon relaxation, the relaxed structure remains very similar to the initial refined structure (these relaxations and change in $G(r)$ occur with a very small energy change, $0.3 \%$ ). Such a loss of long-range order upon relaxation is inherent to any simulation based on reactive force fields. Indeed, there is no existing reactive force field able to describe the bonded $N$-body interactions. The RMC part of the MD-HRMC procedure is therefore necessary in order to impose the long-range order in the structure.

To demonstrate that pure MD simulations cannot reproduce the experimental structure of disordered porous carbons, we performed QMD simulations in which the initial random system is quenched from a temperature of $15000 \mathrm{~K}$ down to $300 \mathrm{~K}$. Varying simulation times were considered to probe the effect of quench rates on the quality of the structures obtained using QMD. The final $G(r)$ functions are compared in Fig. 5(b) with the experimental $G(r)$. This figure shows that a very slow cooling rate ( $>2 \mathrm{~ns}$ ) is necessary to reproduce the long-range order. However, the corresponding molecular configuration for such slow cooling rates shows that the final structure is graphite-like, in contradiction with the known amorphous, non-graphitized structure of such sucrose cokes. ${ }^{15}$ In contrast, for rapid quench rates (10 ps), QMD generates amorphous carbons but their $G(r)$ does not match the experimental target and their structure is highly defective and physically unrealistic. The fact that a structure possesses a structure with a $G(r)$ function similar to that of an experimental sample is an insufficient constraint as there is an infinity of solutions. Such a limitation is illustrated in Fig. 5 which reports three very different structures having the same $G(r)$. In order to produce amorphous structures, we therefore need to use a method that leads to disordered samples, i.e., a method based on a Monte Carlo procedure such as HRMC and other related techniques.

\section{Adsorption properties}

The goal of every reconstruction method is to generate atomistic models of a given sample which are able to reproduce its physical and chemical properties. However, the uniqueness theorem ${ }^{10}$ states that the correspondence between the structure and the $G(r)$ function has a unique solution in the case of pairwise interactions only. In carbonaceous systems, $N$-body interactions are known to play a key-role and have therefore to be taken into account. As pointed out by Palmer and Gubbins ${ }^{4}$ no reactive potential is able to handle the bonded $N$ body interactions, and there exists no experimental target that would gather complete information about the $N$-body system. As a consequence, there is no uniqueness of the solution obtained by HRMC, even though the ensemble of possible configurations is reduced compared to those accessible to a "simple" RMC simulation (Fig. 12 of Ref. 4). The sole ability to reproduce the $G(r)$ function is therefore not sufficient to guarantee that the simulated structure is representative of the real sample. A characterization tool accessible to both simulation and experiment therefore provides additional constraints that are needed to validate molecular models generated using reconstruction techniques. In this regard, experiments probing the microscopic texture of the sample, such as gas adsorption, or its local structure, such as the phonon density of states, Raman and infrared (IR) spectra, and elastic moduli provide important tools to assess the quality of molecular models.

Following previous works, ${ }^{3,27-30}$ Grand canonical Monte Carlo (GCMC) simulations were used to simulate nitrogen adsorption at $77.4 \mathrm{~K}$ in the various samples shown in Fig. 2. The nitrogen molecules are described as a single LennardJones sphere. ${ }^{27}$ Such GCMC simulations also allow determining the isosteric heat of adsorption, which corresponds to the heat $q_{s t}$ released upon adsorption of one mole of adsorbate. In these simulations, $q_{s t}$ is computed from the cross-fluctuations over the number of adsorbed molecules and the total energy ${ }^{3}$ $q_{s t}=R T-\frac{\partial\langle U\rangle}{\partial\langle N\rangle}=R T-\frac{\langle U N\rangle-\langle U\rangle\langle N\rangle}{\left\langle N^{2}\right\rangle-\langle N\rangle^{2}}$. 
Fig. 6 shows that, despite the presence of numerous defects in the HRMC structures, both $n_{a d s}$ and $q_{s t}$ are very similar for all samples. Such adsorption isotherms, which resemble the Langmuir adsorption isotherm, are usually observed for microporous materials where no condensation occurs due to the small size of the pores. ${ }^{3}$ At low pressure, $n_{\text {ads }}$ increases while $q_{s t}$ decreases as the strongly adsorbing sites get filled. Once the pores are filled, $n_{a d s}$ and $q_{s t}$ reach a plateau $q_{s t} \sim 10 \mathrm{~kJ} / \mathrm{mol}$ which is significantly larger than the heat of vaporization of $\mathrm{N}_{2}(5.56 \mathrm{~kJ} / \mathrm{mol})$; this means that the pores are so small that the $\mathrm{N}_{2}$ molecules always interact with the pore surface. These GCMC simulations show that highly energetic defects such as 3- or 4-membered rings do not affect $\mathrm{N}_{2}$ adsorption in their porosity. This result shows that the experimental adsorption properties are a necessary but non-sufficient constraint to develop a realistic model of porous carbons.

\section{Vibrational properties}

In contrast to adsorption properties, defects in porous carbons are expected to significantly affect their vibrational properties. Since they do not contain any information on the local charges, the REBO-based force fields are not suited to properly study the vibrational properties of the structures. In contrast, semi-ab initio force fields such as the Condensedphase Optimized Molecular Potential for Atomistic Simulations Studies (COMPASS) ${ }^{31}$ have proven to be valuable for computing the vibrational properties of carbonaceous systems.

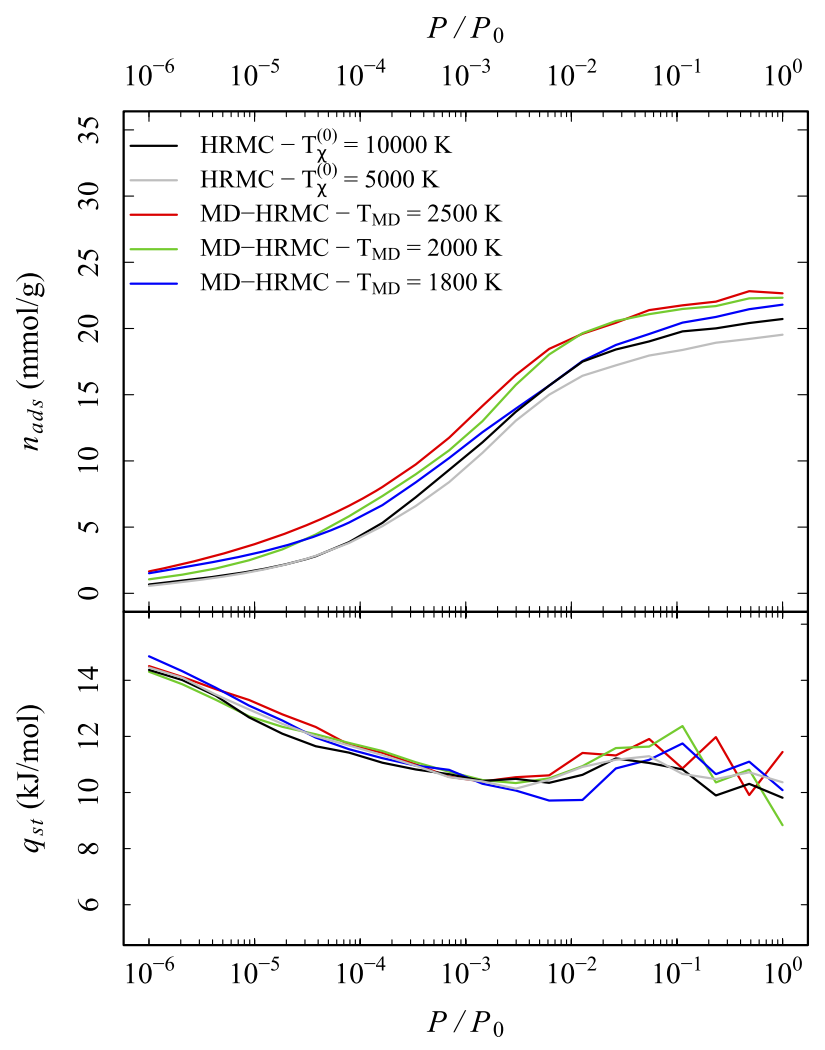

FIG. 6. Simulated $\mathrm{N}_{2}$ adsorption isotherms, $n_{\text {ads }}\left(P / P_{0}\right)$ (top), and isosteric heats of adsorption, $q_{s t}\left(P / P_{0}\right)$ (bottom), for the various samples shown in Fig. 2.
However, their use requires the knowledge of the hybridization state of each atom, which is not available after HRMC refinement since a reactive force field was used. Therefore, in order to assess the vibrational properties of our molecular models, we performed MD relaxations with the ReaxFF force field, ${ }^{32}$ a bond order reactive force field that allows charge equilibration. The phonon DOS is proportional to the Fourier transform of the velocity autocorrelation function (VACF) in the framework of the linear response theory. ${ }^{33,34}$ Fig. 7 shows the DOS of the various samples obtained in this work, together with the DOS of a perfect graphene sheet for comparison. Here, only the carbon-carbon correlations are taken into account.

Fig. 7 shows that despite an overestimation by $\sim 120 \mathrm{~cm}^{-1}$, the forcefield reproduces the characteristic $\mathrm{G}$ band of graphene at $\sim 1583 \mathrm{~cm}^{-1}, 35$ band that is present in all samples and typical of aromatic $\mathrm{sp}^{2}$ carbons. The three most defective samples, i.e., HRMC 10000 , HRMC 5000, and MD-HRMC 1800, show a smaller and broader G-like band compared to less defective samples, MD-HRMC 2000 and 2500: such a broadening of the $G$ band results from the numerous defects inducing a distribution of D-like modes.

The results above confirm that, in contrast to adsorption, the vibrational properties of a structure are highly sensitive to defects. This suggests that the comparison between experimental and simulated phonon densities of states is a powerful tool for validating reconstructed structures. Other spectroscopic techniques such as Raman and IR spectroscopies can be used to assess the quality and validity of the local chemistry of the reconstructed molecular models. IR and Raman spectra can either be computed in a classical way ${ }^{34,36}$ or using $a b$ initio quantum calculations with available packages such as CASTEP $^{37}$ or VASP. ${ }^{38}$ Computing the elastic properties of the refined structures could also constitute a conclusive test regarding their validity. However, special attention should be paid to finite size effects that can occur when dealing with small simulation boxes. Finally, in dense systems, adsorption measurements are hampered by pore accessibility issues and measured pore size distribution, and specific surface areas should be considered with caution. In contrast, comparison

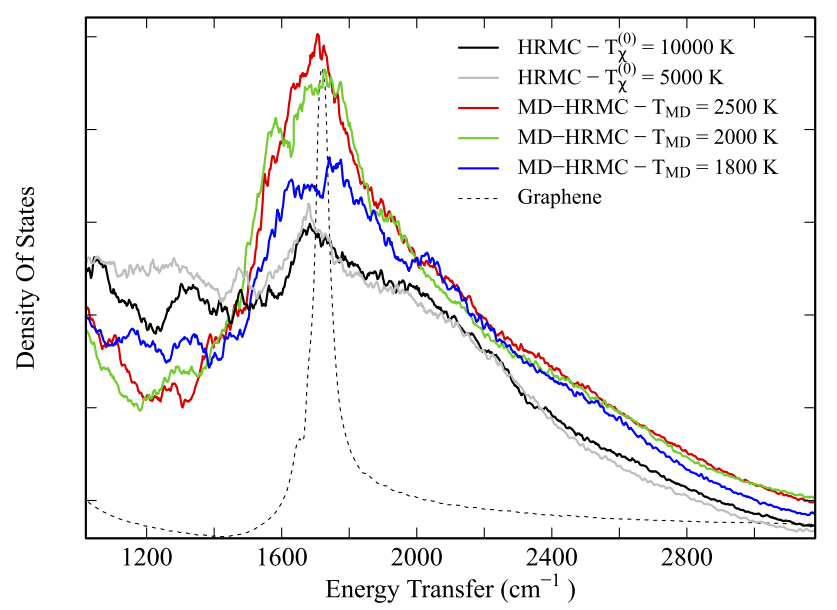

FIG. 7. Simulated phonon densities of states (DOS) at $300 \mathrm{~K}$ for the various samples shown in Fig. 2. 
between quasi-elastic neutron scattering (QENS) and diffusion simulation ${ }^{39,40}$ constitute a powerful tool to validate the texture of reconstructed molecular models.

\section{CONCLUSION}

An improved and accelerated version of the HRMC is proposed by combining this reconstruction technique with MD. We have also proposed a rational choice of the various parameters in these simulations in order to make the technique transferable. Such a MD-HRMC method is at least one order of magnitude faster than HRMC for obtaining structures of similar quality. This method, which has been applied to disordered porous carbons, can be easily transferred to other systems provided that a reactive force field describing the interactions in the system is available. In particular, while the results were obtained here with the REBO forcefield, the increase in computational speed for the MD-HRMC method is not related to the specific choice of the interatomic potential but linked to the addition of MD relaxation steps.

We have also provided several tools for testing the validity and the quality of the refined structures. On the one hand, the adsorption properties test the global texture of the sample but they are not sensitive to defects. This shows that the adsorption properties constitute a necessary but insufficient characterization test. On the other hand, the vibrational properties (such as the phonon density of states or the Raman/IR spectrum) are highly sensitive to the local structure. Their comparison to experimental data therefore constitutes a strong constraint on the refined model.

\section{ACKNOWLEDGMENTS}

This work has been carried out within the framework of the ICoME2 Labex (No. ANR-11-LABX-0053) and the A* MIDEX projects (No. ANR-11-IDEX-0001-02) co-funded by the French program "Investissements d'Avenir" which is managed by the ANR, the French National Research Agency. We also acknowledge funding from Royal Dutch Shell and Schlumberger through the MIT X-Shale research project.

${ }^{1}$ M. E. Davis, Nature 417, 813 (2002).

${ }^{2}$ L. D. Gelb, MRS Bull. 34, 592 (2009).

${ }^{3}$ B. Coasne, A. Galarneau, R. J. Pellenq, and F. Di Renzo, Chem. Soc. Rev. 42, 4141 (2013).
${ }^{4}$ J. C. Palmer and K. E. Gubbins, Microporous Mesoporous Mater. 154, 24 (2012).

${ }^{5}$ F. R. Siperstein and K. E. Gubbins, Mol. Simul. 27, 339 (2001).

${ }^{6}$ J. Tersoff, Phys. Rev. Lett. 61, 2879 (1988).

${ }^{7}$ R. McGreevy and L. Pusztai, Mol. Simul. 1, 359 (1988).

${ }^{8}$ R. McGreevy, Nucl. Instrum. Methods Phys. Res., Sect. A 354, 1 (1995).

${ }^{9}$ N. Metropolis, A. W. Rosenbluth, M. N. Rosenbluth, A. H. Teller, and E. Teller, J. Chem. Phys. 21, 1087 (1953).

${ }^{10}$ R. Henderson, Phys. Lett. A 49, 197 (1974).

${ }^{11}$ R. McGreevy, J. Phys.: Condens. Matter 3, F9 (1991).

${ }^{12}$ K. T. Thomson and K. E. Gubbins, Langmuir 16, 5761 (2000).

${ }^{13}$ D. A. Keen, Phase Transitions 61, 109 (1997).

${ }^{14}$ P. Biswas, R. Atta-Fynn, and D. A. Drabold, Phys. Rev. B 69, 195207 (2004).

${ }^{15}$ J. Pikunic, C. Clinard, N. Cohaut, K. E. Gubbins, J.-M. Guet, R. J.-M. Pellenq, I. Rannou, and J.-N. Rouzaud, Langmuir 19, 8565 (2003).

${ }^{16}$ J. Pikunic, P. Llewellyn, R. J.-M. Pellenq, and K. E. Gubbins, Langmuir 21, 4431 (2005).

${ }^{17}$ S. Jain, J. Fuhr, R.-M. Pellenq, J. Pikunic, C. Bichara, and K. Gubbins, Studies in Surface Science and Catalysis (Elsevier, 2006), Vol. 160, pp. 169-176; Stud. Surf. Sci. Catal. 160, 169 (2007).

${ }^{18}$ S. K. Jain, "Molecular modeling of microporous and templated mesoporous carbons," Ph.D. thesis (North Carolina State University, Raleigh, 2008).

${ }^{19}$ G. Opletal, T. Petersen, B. O’Malley, I. Snook, D. G. McCulloch, N. A. Marks, and I. Yarovsky, Mol. Simul. 28, 927 (2002).

${ }^{20}$ O. Gereben and L. Pusztai, J. Comput. Chem. 33, 2285 (2012).

${ }^{21}$ S. Jain, R. Pellenq, J. Pikunic, and K. Gubbins, Langmuir 22, 9942 (2006).

${ }^{22}$ T. Nguyen, S. Bhatia, S. Jain, and K. Gubbins, Mol. Simul. 32, 567 (2006).

${ }^{23}$ P. Biswas, D. N. Tafen, and D. A. Drabold, Phys. Rev. B 71, 054204 (2005).

${ }^{24}$ S. K. Jain, J. P. Pikunic, R. J.-M. Pellenq, and K. E. Gubbins, Adsorption 11, 355 (2005).

${ }^{25}$ D. W. Brenner, O. A. Shenderova, J. A. Harrison, S. J. Stuart, B. Ni, and S. B. Sinnott, J. Phys.: Condens. Matter 14, 783 (2002).

${ }^{26}$ S. Plimpton, J. Comput. Phys. 117, 1 (1995).

${ }^{27}$ P. Billemont, B. Coasne, and G. De Weireld, Langmuir 29, 3328 (2013).

${ }^{28}$ B. Coasne, K. E. Gubbins, F. R. Hung, and S. K. Jain, Mol. Simul. 32, 557 (2006).

${ }^{29}$ B. Coasne, S. Jain, and K. Gubbins, Mol. Phys. 104, 3491 (2006).

${ }^{30}$ S. Jain, K. Gubbins, R. J.-M. Pellenq, and J. Pikunic, Carbon 44, 2445 (2006).

${ }^{31}$ H. Sun, J. Phys. Chem. B 102, 7338 (1998).

${ }^{32}$ A. C. van Duin, S. Dasgupta, F. Lorant, and W. A. Goddard, J. Phys. Chem. A 105, 9396 (2001).

${ }^{33}$ G. Squires, Thermal Neutron Scattering (Cambridge University Press, Cambridge, 1978).

${ }^{34}$ M. Thomas, M. Brehm, R. Fligg, P. Vöhringer, and B. Kirchner, Phys. Chem. Chem. Phys. 15, 6608 (2013).

${ }^{35}$ L. Malard, M. Pimenta, G. Dresselhaus, and M. Dresselhaus, Phys. Rep. 473, 51 (2009).

${ }^{36}$ M. Ricci, G. Signorelli, and V. Mazzacurati, J. Phys.: Condens. Matter 2, 19 (1990).

${ }^{37}$ S. J. Clark, M. D. Segall, C. J. Pickard, P. J. Hasnip, M. I. Probert, K. Refson, and M. C. Payne, Z. Kristallogr. 220, 567 (2005).

${ }^{38}$ G. Kresse and J. Hafner, Phys. Rev. B 47, 558 (1993).

${ }^{39}$ A. H. Farmahini, A. Shahtalebi, H. Jobic, and S. K. Bhatia, J. Phys. Chem. C 118, 11784 (2014).

${ }^{40}$ A. Shahtalebi, A. H. Farmahini, P. Shukla, and S. K. Bhatia, Carbon 77, 560 (2014). 